\title{
11q22.2q22.3 microdeletion syndrome
}

INSERM

\section{Source}

INSERM. (1999). Orphanet: an online rare disease and orphan drug data base.

11q22.2q22.3 microdeletion syndrome. ORPHA:444002

11 q22.2q22.3 microdeletion syndrome is a rare chromosomal anomaly characterized by mild intellectual disability, developmental delay, short stature, hypotonia and dysmorphic facial features. Anxiety and short attention span have also been reported. 